BMJ Open

Diabetes

Research

\& Care

\title{
Serum galectin-3BP as a novel marker of obesity and metabolic syndrome in Chinese adolescents
}

\author{
Shihan Zhen (D , , Yanan Ma, ${ }^{2}$ Yanshuo Han, ${ }^{2}$ Zhongyi Zhao, ${ }^{2}$ Xuelian Yang, ${ }^{1}$ \\ Deliang Wen ${ }^{1}$
}

To cite: Zhen S, Ma Y, Han Y, et al. Serum galectin-3BP as a novel marker of obesity and metabolic syndrome in Chinese adolescents. BMJ Open Diab Res Care 2021;9:e001894. doi:10.1136/ bmjdrc-2020-001894

- Supplemental material is published online only. To view, please visit the journal online (http://dx.doi.org/10.1136/ bmjdrc-2020-001894).

Received 9 September 2020 Revised 1 February 2021 Accepted 7 February 2021
Check for updates

(C) Author(s) (or their employer(s)) 2021. Re-use permitted under CC BY-NC. No commercial re-use. See rights and permissions. Published by BMJ.

${ }^{1}$ Institute of Health Sciences, China Medical University, Shenyang, China

${ }^{2}$ School of Public Health, China Medical University, Shenyang, China

Correspondence to Dr Deliang Wen; dlwen@cmu.edu.cn

\section{ABSTRACT}

Introduction Childhood obesity (OB) and metabolic syndrome (MetS) have become a worldwide health problem. Comparative proteomic approaches are widely used in human $\mathrm{OB}$ to analyze protein changes in blood plasma. The present study determined the galectin-3 binding protein (galectin-3BP) expression level in different weight categories and assessed the associations between galectin-3BP and $\mathrm{OB}$ and MetS.

Research design and methods The current study included 932 Chinese adolescents 13-18 years of age. The biochemical and anthropometric variables of all the subjects were evaluated using standardized procedures. The differentially expressed proteins (DEPs) were investigated among 60 adolescents (20 normal weight, 20 overweight and 20 obese) using tandem mass tag (TMT) quantitative proteomics. The serum galectin-3BP level was measured using ELISA. The associations between galectin$3 \mathrm{BP}$ and $\mathrm{OB}$ and MetS were analyzed in 932 adolescents using multiple logistic regression analyses.

Results A significant DEP, galectin-3BP, can effectively separate the obese from the normal weight group using TMT. Adolescents in tertile 3 of galectin-3BP, when compared with adolescents in the tertile 1 , were positively associated with $\mathrm{OB}(\mathrm{OR}=3.32,95 \% \mathrm{Cl} 1.79$ to 6.16$)$ and MetS $(\mathrm{OR}=3.28,95 \% \mathrm{Cl} 1.30$ to 8.26$)$. The receiver operating characteristic curve for galectin-3BP in subjects with MetS indicated that the area under the curve was 0.85 (95\% Cl 0.79 to 0.91 ).

Conclusions This study confirmed an association between galectin-3BP and $\mathrm{OB}$ in Chinese adolescents, and galectin-3BP was also positively associated with MetS, and thus might be useful for identifying adolescents with MetS.

\section{INTRODUCTION}

Childhood obesity (OB) and metabolic syndrome (MetS) have become a worldwide health problem. The percentage of overweight and obese children has increased at an alarming rate. Between 1991 and 2014, the prevalence of overweight (OW) in Chinese children continuously increased from $5.0 \%$ to $13.2 \%$, while the prevalence to $\mathrm{OB}$ increased from $1.7 \%$ to $6.8 \% .^{12}$ These large increases in the prevalence of childhood OB might greatly increase morbidity in adulthood from MetS. ${ }^{3}$ MetS is a group of complex metabolic disorders

\section{Significance of this study}

What is already known about this subject?

- Childhood obesity (OB) and metabolic syndrome (MetS) have become a worldwide health problem, and comparative proteomic approaches are widely used in human $\mathrm{OB}$ to analyze protein changes in blood plasma.

What are the new findings?

- When comparing the proteomes among normal weight, overweight and obese subjects, galectin-3 binding protein (galectin-3BP) was a potential hepatic metabolic biomarker.

- There were significant positive associations between galectin-3BP expression and $\mathrm{OB}$ and MetS in Chinese adolescents.

How might these results change the focus of research or clinical practice?

- For the prevention of MetS, clinicians can focus on the serum galectin-3BP level.

that affect carbohydrates, proteins, fats, and other substances in the body. ${ }^{4}$ As reported in a 2015 meta-analysis, MetS has an incidence of $1.8 \%-4.5 \%$ in Chinese adolescents; more specifically, the incidence follows the order of $\mathrm{OB}>\mathrm{OW}>$ normal weight $(\mathrm{NW}) .^{5}$ Typically, an increase in MetS incidence increases morbidities, including cancers, arthritis, diabetes, and cardiovascular diseases. ${ }^{6} 7$ Therefore, it is essential to identify biomarkers to predict MetS risk among adolescents, which in turn will facilitate improved management of MetSassociated public health problems.

Tandem mass tag (TMT) is a protein quantitative technique based on tandem mass spectrometry that is widely used in screening the biomarkers for prognosis and diagnosis. ${ }^{89}$ TMT has been used to analyze the proteomic changes of obese individuals. ${ }^{8}$ Consequently, the clinical proteomic strategy facilitates a comprehensive understanding of serum factors that are involved in childhood $\mathrm{OB}$ 
and sheds light on changes in the signal transduction pathways. Determining the proteomic changes in childhood OB may contribute to a better understanding of the pathogenesis underlying MetS. Specifically, serum proteomic analysis serves as an efficient way to identify protein biomarkers to recognize, diagnose, monitor and treat various disorders, such as $\mathrm{OB}$ and MetS. ${ }^{1011}$

Galectin-3 binding protein (galectin-3BP), also referred to as M2BP, is a largely glycosylated protein containing seven N-linked glycosylation sites. ${ }^{12}$ Galectin-3BP, which has multimeric forms, has several targets, such as galectin-1, galectin-3, galectin-7 and extracellular matrix. ${ }^{12} 13$ The full complement of galectin-3BP biological activities is not known, but it is known that galectin-3BP participates in cell growth, inflammation, cellular adhesion, and an increase in visceral fat deposition. ${ }^{13-15}$ Additionally, it has been reported that galectin-3BP expression is detected among different cell types, such as mucosal or glandular epithelium and hematopoietic cells $^{1617}$; galectin-3BP is highly expressed within serum in patients with hepatitis, non-alcoholic fatty liver disease, and autoimmune disease. ${ }^{15} 17$ Plasma galectin-3BP has been reported to be elevated in obese individuals ${ }^{1819}$ and individuals with symptoms of MetS. ${ }^{19-21}$ To the best of our knowledge, however, most previous studies involved adults; the variations in galectin-3BP expression among obese adolescents or adolescents with MetS have rarely been reported.

Although several studies have reported increased levels of galectin-3BP in the obese individuals, ${ }^{18} 19$ few studies have investigated the relationship in adolescents. The association between galectin-3BP and MetS has not been established. By adopting the proteomic method in a wellcharacterized Chinese study, we investigated the differentially expressed proteins (DEPs) among adolescents with NW, OW, and $\mathrm{OB}$, and investigated the associations between galectin-3BP and $\mathrm{OB}$ and MetS.

\section{METHODS}

\section{Clinical information}

An observational, school-based, case-control study was conducted to estimate OB from a multicenter prospective cohort (Huanggu District Middle and Primary School Student Physical Fitness Monitoring). ${ }^{22}$ This cohort was established to investigate the health outcomes, weight status, and growth status, and to explore the geneticenvironmental interactions of primary and middleschool children and adolescents in Northeast China. For TMT quantitative proteomics, a total of 60 representative adolescents meeting those predetermined inclusion criteria were enrolled. Then, 20 samples from each group that had the equivalent serum volumes were blended. Subsequently, proteins with high abundance were depleted from the resulting mixed serum samples. After anthropometry measurements and blood sample analyses were completed, a total of 932 adolescent students (13-18 years of age) were eligible to participate. The participants were used to verify the reliability of TMT. A flowchart showing the participant selection process is provided in online supplemental figure S1.

Weight and height were determined in accordance with the WHO recommended normalized techniques. In addition, adolescents with $\mathrm{OB}$ and $\mathrm{OW}$ were determined based on their sex and age in accordance with the Body Mass Index (BMI) growth references for 5-19 years formulated by the WHO. MetS was diagnosed in accordance with the Society of Pediatrics of Chinese Medical Association report on MetS in the adolescent population. ${ }^{23}$ Participants and their parents had provided written informed consent to perform venipuncture and were informed about the intended use of the sample.

\section{TMT labeling and grouping}

TMT labeling reagent $(20 \mu \mathrm{L})$ was added to every peptide solution for 1 hour of reaction. Typically, peptide was labeled using a TMT 10-plex kit (Thermo Fisher Scientific, Waltham, Massachusetts, USA) in the TMT 10-plex Label Reagent Set in accordance with manufacturer's instructions.

Sixty samples were randomized into six groups for liquid chromatography with tandem mass spectrometry and TMT analyses. The control participants had matched sex, ethnicity, and maternal age (online supplemental figure S1). Peptides from the adolescent with $\mathrm{OB}$, OW and NW were labeled using TMT-126, TMT-127N, TMT127C, TMT-128N, TMT-128C and TMT-129N reagents (TMT; Thermo, Pierce Biotechnology, Rockford, Illinois, USA).

\section{Data analyses}

Workflow of Proteome Discoverer software V.1.3 (Thermo Fisher Scientific, San Jose, California, USA) was used to process all mass spectrometry (MS) information. Thereafter, the Proteome Discoverer extract feature was used to process those data about tandem mass spectrometry spectra using the MASCOT retrieval function in the workflow. In addition, a human database (201709, time files compressed): Tue Nov 28 15:06:52 2017) containing 71591 sequences was adopted to retrieve those tandem mass spectra.

The Proteome Discoverer automatically analyzes results statistically, which also adopts the unique peptides for accurately calculating the relative protein level. The top one peptide rank filters and high peptide confidence were employed to extract protein and peptide data. In addition, the decoy database was used to analyze peptide sequences, so as to calculate the false discovery rate (FDR). Then, the percentage variability and the mean ratio were adopted to quantify proteins in which several peptides were found within a single protein.

\section{Functional analyses for those DEPS}

The UniProt-GOA database was used to perform the analysis. Specifically, there are three Gene Ontology (GO) annotation classes, including molecular function (MF), cellular compartment (CC) as well as biological process (BP). In 
every category, DEP enrichment was tested through the two-tailed Fisher's exact test among the proteins identified. The normalized FDR control approach was used to correct multiple hypothesis testing, and those GO terms that had an adjusted $p$ value of $<0.05$ were considered significant.

In addition, protein pathways were annotated using the Kyoto Encyclopedia of Genes and Genomes (KEGG) database. First of all, KAAS, the online service tool of KEGG, was used to annotate protein descriptions in the KEGG database. Then, the annotation results were mapped onto the KEGG database with the KEGG mapper, another online service approach of KEGG.

\section{Biomarker verification}

Human galectin-3BP ELISA Kit (EK1240, Cambridge, Ontario, Canada) was used to examine galectin-3BP through the ELISA strictly following manufacturer protocols. In addition, the original standards provided by the kit were adopted to dilute gradually, and biomarker concentrations within specific samples were calculated using standard curves. Besides, the serum galectin-3BP (dilution, 1:500) was detected through the human galectin-3BP ELISA kit, and the minimal detectable dose was $156 \mathrm{pg} / \mathrm{mL}$. Each sample was tested in duplicate.

\section{Definition of the galectin-3BP group}

The participants were divided into three groups according to the tertiles of the galectin-3BP concentration. Participants were divided into 'tertile 1', 'tertile 2' and 'tertile
3' groups. An increased concentration was assumed from tertiles 1-3.

\section{Statistical analysis}

The relative expression levels of protein expression among adolescents with $\mathrm{OB}, \mathrm{OW}$, and NW were compared with identified DEPs. Specifically, proteins were deemed to show differential expression when TMT ratios in the OB or OW serum samples were $<0.67$ or $>1.5$ in comparison with adolescents with NW.

Descriptive information was presented in mean and SD for Gaussian distribution and medians together with the upper and lower quartiles for non-Gaussian distribution. Multiple logistic regression model was used to determine the relationships between galectin-3BP and weight status and MetS. Then, logistic regression modeling and the receiver operating characteristic (ROC) curves were used to jointly detect candidate biomarkers and analyze the individual roles. A p value of $<0.05$ indicated statistical significance. In the current work, both SPSS V.25.0 and STATA V.13.0 were used to analyze statistically. Meanwhile, R Studio (R Studio, USA) and GraphPad Prism V.5.0 (GraphPad, La Jolla, California, USA) were used for graph preparation.

\section{RESULTS}

Participant characteristics and protein identification based on TMT

A total of 60 serum samples were collected from adolescents with $\mathrm{OB}$, OW, and NW matched for sex and age

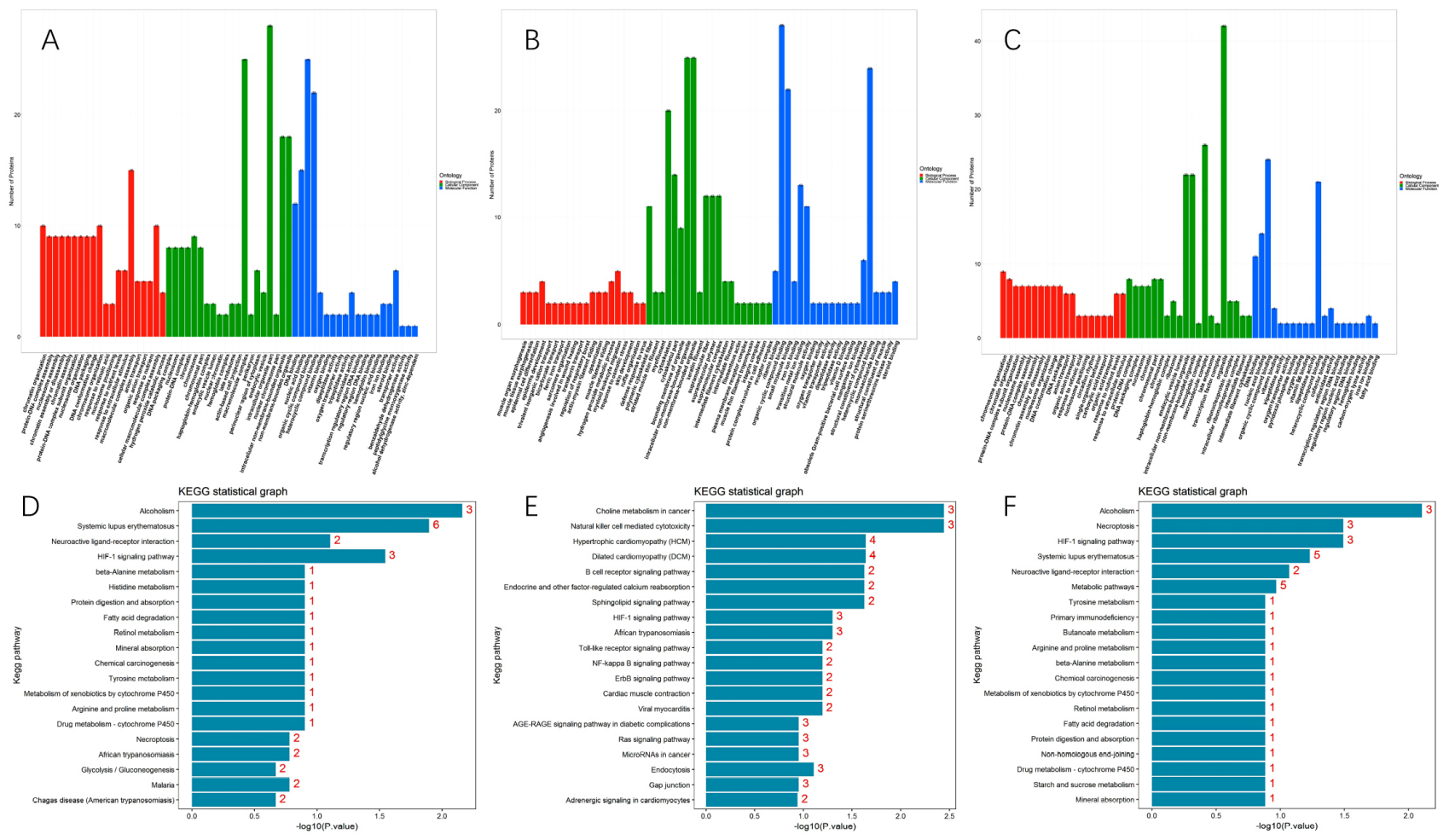

Figure 1 Detailed functional classifications of the identified proteins. (A-C) GO analysis was performed to identify the functional significance for each screened protein. (D-F) KEGG pathway analysis was used to elucidate the enrichment pathway. GO, Gene Ontology; KEGG, Kyoto Encyclopedia of Genes and Genomes. 
in the current work (online supplemental table S1). Online supplemental figure S2 shows validation of MS data. In total, 680 proteins were examined using TMT MS analyses on serum samples among adolescents with different weight states (online supplemental figure S3). In the current work, 177 proteins that had an average fold change in expression level of $> \pm 1.5$ were recognized to be the DEPs. Among the 177 proteins, 85 proteins were shown to be significantly differentially expressed in OW samples relative to NW. The DEPs were selected in the current work to effectively separate the OB and OW groups from the NW group. Additionally, results of hierarchical clustering proved the reasonability of the as-screened DEPs.

\section{Annotation analyses for DEPs ( $\mathrm{GO}+\mathrm{KEGG)}$}

GO analysis was conducted to identify the functional significance for each screened protein. Figure 1A-C presents the functional classifications for each as-screened protein. Unlike the as-screened proteins, a few of the proteins $(n<10)$ were associated with the BP classifications of chromosome organization $(n=9)$ and chromatin organization $(n=8)$ in the adolescents with OB compared with the adolescents with NW. In the cellular protein classification, organelle $(n=42)$, macromolecular complex $(n=26)$, intracellular organelle with no membrane binding $(n=22)$, and organelle with no membrane binding $(n=22)$ were the major CCs. For the MF classification, heterocyclic compound binding $(n=21)$ and nucleic acid binding $(\mathrm{n}=14)$ were the primary functional categories for those reduced proteins (figure 1C).

According to KEGG pathway analysis, a total of 69,146 and $69 \mathrm{KEGG}$ pathways were clustered for the OW/NW, $\mathrm{OB} / \mathrm{OW}$, and $\mathrm{OB} / \mathrm{NW}$ groups, respectively. Figure 1D-F provided a panoramic view of the systemic lupus erythematosus (SLE) pathway, the metabolic pathway, the hypertrophic cardiomyopathy (HCM) pathway, and the dilated cardiomyopathy (DCM) pathway, and they were primarily involved DEPs. Relative to the NW group, the elevated proteins in adolescents with $\mathrm{OB}$ were mainly involved in the SLE (5) and metabolic pathway (5) in figure $1 \mathrm{~F}$, whereas compared with the $\mathrm{OW}$ group, the elevated proteins of the adolescents with $\mathrm{OB}$ were mainly involved in the HCM (4) and DCM (4) pathways, as shown in figure $1 \mathrm{E}$.

\section{Verification of the differential expression of galectin-3BP}

According to the intersection analysis within three comparative groups through hierarchal clustering analysis (figure 2) and KEGG network analysis, there were altogether 26 DEPs (online supplemental table S2). The aforementioned findings were synthesized, such as the ratio of fold change, as well as KEGG and GO analysis, and it was shown that, a majority of significant DEPs were related to the metabolic pathway.

Moreover, the intersection analysis identified 26 potential proteins for hierarchal clustering analysis, 11 of which were excluded because they were not involved in the elevated proteins. The serum levels of serum amyloid A, $\mathrm{C}$ reactive protein, plasminogen activator inhibitor-1, and alcohol dehydrogenase ${ }^{24}{ }^{25}$ have been previously investigated in children or adolescents with $\mathrm{OB}$. Another three proteins were identified to contain the non-gradient change among the three weight groups, including two overlapping with the included proteins from the same description, two forming different cytoskeletal types, and one belonging to the protein fragment. Moreover, the galectin-3BP was selected to carry out further study, since neither charged multivesicular body protein 6 nor inhibin beta $\mathrm{E}$ chain commercial kit was available. Figure 2 presents flowcharts to obtain these in the trial.

\section{Associations between galectin-3BP and OB or MetS}

Table 1 shows the feature for the whole participants. The participants were classified based on the tertile for

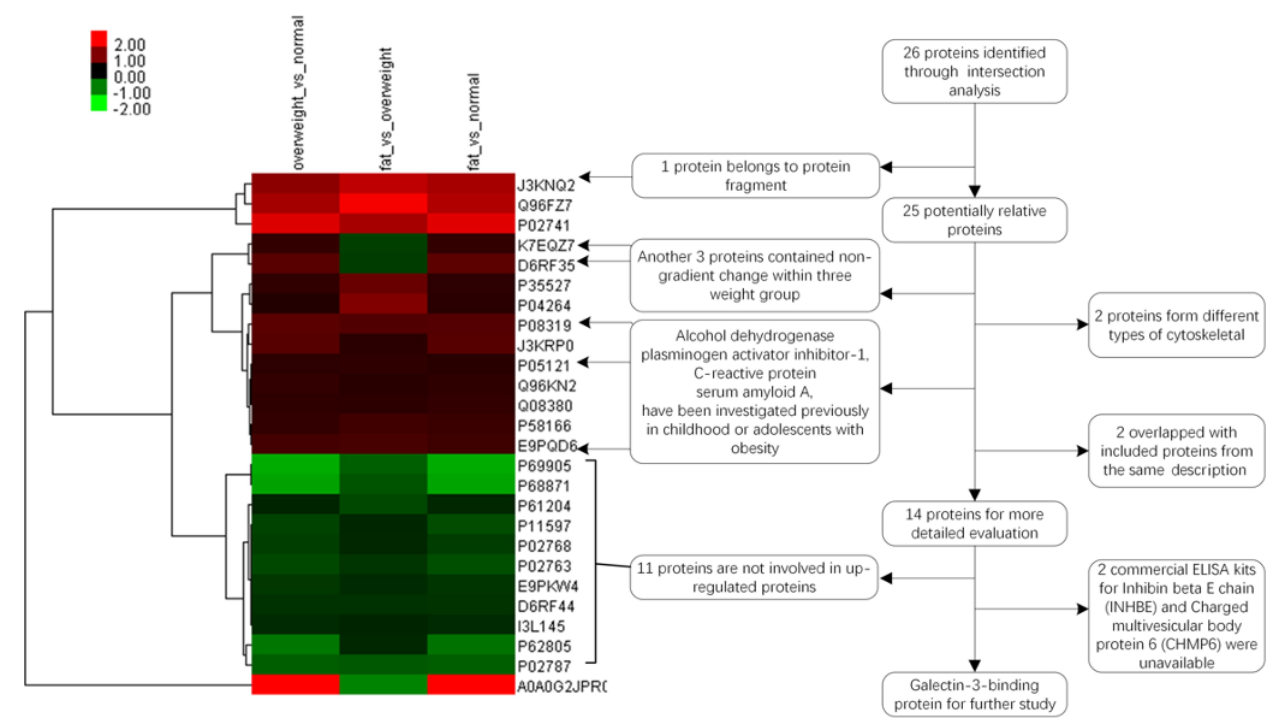

Figure 2 Flowcharts to obtain intersection in the trial: the 26 protein expressions in 60 adolescents' plasma and the screening details of protein based on intersection analysis. 
Table 1 Characteristics of the study population according to tertiles of galectin-3BP level

\begin{tabular}{|c|c|c|c|}
\hline Characteristic & Tertile $1(n=310)$ & Tertile $2(n=311)$ & Tertile $3(n=311)$ \\
\hline Age (years), mean (SD) & $16.27(1.04)$ & $16.27(1.02)$ & $16.05(0.91)$ \\
\hline Girls, n (\%) & $144(46.45)$ & $148(47.59)$ & $190(61.09)$ \\
\hline \multicolumn{4}{|l|}{ Anthropometry } \\
\hline BMl z-score, mean (SD) & $0.06(1.17)$ & $0.43(1.36)$ & $0.65(1.36)$ \\
\hline Waist circumference, mean (SD) & $71.95(8.60)$ & $75.00(11.54)$ & $75.97(12.01)$ \\
\hline \multicolumn{4}{|l|}{ Weight status, n (\%) } \\
\hline NW & $250(80.65)$ & $204(65.59)$ & $181(58.20)$ \\
\hline OW & $40(12.90)$ & $61(19.61)$ & $73(23.47)$ \\
\hline OB & $20(6.45)$ & $46(14.79)$ & $57(18.33)$ \\
\hline \multicolumn{4}{|l|}{ MetS outcomes } \\
\hline MetS, n (\%) & $8(2.58)$ & $15(4.82)$ & $23(7.40)$ \\
\hline Center obesity, n (\%) & $48(15.48)$ & $92(29.58)$ & 106 (34.08) \\
\hline Hypertension, n (\%) & $69(22.26)$ & $66(21.22)$ & $82(26.37)$ \\
\hline Hyperglycemia, n (\%) & $3(0.97)$ & $5(1.61)$ & $5(1.61)$ \\
\hline High_TG, n (\%) & 26 (8.39) & $30(9.65)$ & $26(8.36)$ \\
\hline Low_HDL-C, n (\%) & $35(11.29)$ & $43(13.83)$ & $48(15.43)$ \\
\hline \multicolumn{4}{|l|}{ Laboratory examinations } \\
\hline FPG (mmol/L), median (Q1-Q3) & $4.28(4.0-4.56)$ & $4.25(3.96-4.53)$ & $4.31(4.05-4.6)$ \\
\hline TG (mmol/L), median (Q1-Q3) & $0.71(0.53-0.98)$ & $0.76(0.55-1.04)$ & $0.80(0.59-1.07)$ \\
\hline ALT (U/L), median (Q1-Q3) & $10(8-16)$ & $10(8-17)$ & $10(8-17)$ \\
\hline AST (U/L), median (Q1-Q3) & $15(13-18)$ & $16(13-19)$ & $15(13-18)$ \\
\hline ALP (U/L), median (Q1-Q3) & $96(76-126)$ & $93(77-117)$ & $93(74-126)$ \\
\hline GGT (U/L), median (Q1-Q3) & $16(13-20)$ & $16(13-22)$ & $16(13-22)$ \\
\hline C1q (mg/L), median (Q1-Q3) & $176.0(156.6-201.4)$ & $177.6(154.6-200.2)$ & $184.5(167.5-209.5)$ \\
\hline HDL-C (mmol/L), median (Q1-Q3) & $1.31(1.14-1.50)$ & $1.30(1.11-1.51)$ & $1.28(1.13-1.51)$ \\
\hline LDL-C (mmol/L), median (Q1-Q3) & $2.08(1.70-2.50)$ & $2.12(1.79-2.52)$ & $2.19(1.80-2.58)$ \\
\hline Apolipoprotein A1 (g/L), median (Q1-Q3) & $1.30(1.21-1.42)$ & $1.31(1.20-1.41)$ & $1.32(1.19-1.45)$ \\
\hline Apolipoprotein B (g/L), median (Q1-Q3) & $0.61(0.52-0.72)$ & $0.63(0.54-0.73)$ & $0.66(0.56-0.76)$ \\
\hline $\begin{array}{l}\text { Small dense low-density lipoprotein cholesterol } \\
\text { (mmol/L), median (Q1-Q3) }\end{array}$ & $0.42(0.33-0.52)$ & $0.42(0.33-0.51)$ & $0.43(0.34-0.53)$ \\
\hline
\end{tabular}

ALP, alkaline phosphatase; ALT, alanine transaminase; AST, aspartate aminotransferase; BMI, Body Mass Index; FPG, fasting plasma glucose; galectin-3BP, galectin-3 binding protein; GGT, gamma-glutamyl transferase; HDL-C, high-density lipoprotein cholesterol; LDL-C, low-density lipoprotein cholesterol; MetS, metabolic syndrome; NW, normal weight; OB, obesity; OW, overweight; TG, triglyceride.

galectin-3BP expression. Participants in the highest tertile of galectin-3BP were more likely to be $\mathrm{OB}$ and MetS.

After adjusting for confounders, adolescents in tertiles 3 and 2 of the galectin-3BP level had higher odds of having $\mathrm{OB}$ than adolescents in tertile $1(\mathrm{OR}=3.32,95 \% \mathrm{CI}$ 1.79 to 6.16 for tertile 3 ; OR=2.48, $95 \%$ CI 1.32 to 4.65 for tertile 2), adolescents in tertile 3 of the galectin-3BP level had higher odds of MetS than those in tertile $1(\mathrm{OR}=3.28$, $95 \%$ CI 1.30 to 8.26 for tertile 3 ). The results are shown in table 2. The area under the curve was 0.85 (95\% CI 0.79 to 0.91 ). The results are shown in figure 3 .

\section{DISCUSSION}

This study examined the heterogeneity of protein expression among the Chinese adolescents with or without OB/
MetS, and to validate galectin-3BP expression in samples collected from the study population. Galectin-3BP served as a novel biomarker screened to be related to being $\mathrm{OB}$ or having MetS. Indeed, this study suggested that participants in the highest tertile of galectin-3BP level were more likely to be $\mathrm{OB}$ and MetS, and they were consistent with findings obtained from prior research on adults. ${ }^{18} 19$

Galectins have been shown to be essential in regulating cell growth and adhesion, triggering or inhibiting cell apoptosis, ${ }^{146}$ which may stimulate preadipocytes to differentiate into lipid-loaded adipocytes, enhance the proliferation of adipocytes and result in closer relationship with OB. ${ }^{27}$ Moreover, several animal studies have shown that galectins may serve as the factors to positively regulate OB induced by high-fat diet, and they are increased 
Table 2 Multivariable adjusted ORs and 95\% Cl for OW, OB and MetS across tertiles of galectin-3BP level

\begin{tabular}{|c|c|c|c|c|}
\hline & \multicolumn{3}{|c|}{ Tertiles, OR (95\% Cl) } & \multirow{2}{*}{$\begin{array}{l}\text { P value } \\
\text { for trenc }\end{array}$} \\
\hline & Tertile $1(n=310)$ & Tertile $2(n=311)$ & Tertile $3(n=311)$ & \\
\hline \multicolumn{5}{|l|}{$\mathrm{OW}+\mathrm{OB}$} \\
\hline Age-adjusted model & 1 (reference) & 2.21 (1.53 to 3.19$)$ & 2.87 (2.00 to 4.13$)$ & $<0.001$ \\
\hline Multiple-adjusted model & 1 (reference) & $2.38(1.55$ to 3.67$)$ & 2.95 (1.92 to 4.54$)$ & $<0.001$ \\
\hline \multicolumn{5}{|l|}{ OB } \\
\hline Age-adjusted model & 1 (reference) & 2.53 (1.46 to 4.39$)$ & $3.13(1.83$ to 5.36$)$ & $<0.001$ \\
\hline Multiple-adjusted model & 1 (reference) & $2.48(1.32$ to 4.65$)$ & $3.32(1.79$ to 6.16$)$ & $<0.001$ \\
\hline \multicolumn{5}{|l|}{ MetS } \\
\hline Age-adjusted model & 1 (reference) & $1.92(0.80$ to 4.61$)$ & 2.85 (1.25 to 6.48$)$ & 0.011 \\
\hline Multiple-adjusted model & 1 (reference) & $1.58(0.59$ to 4.24$)$ & 3.28 (1.30 to 8.26$)$ & 0.008 \\
\hline
\end{tabular}

Multiple-adjusted model: adjusted for age (in years), sex (boys vs girls), ALT (U/L), AST (U/L), ALP (U/L), and GGT (U/L). P value for trend was obtained by adjusting tertiles of galectin-3BP level as a continuous variable. $P$ values of $<0.05$ are in bold.

ALP, alkaline phosphatase; ALT, alanine transaminase; AST, aspartate aminotransferase; galectin-3BP, galectin-3 binding protein; GGT, gamma-glutamyl transferase; MetS, metabolic syndrome; OB, obesity; OW, overweight.

in OB and in the differentiation of adipocytes. ${ }^{28}{ }^{29}$ Galectin-3BP is a member of the fucosylated glycoprotein family, which binds with galectin-1, galectin-3, galectin-7 and galectin-9 for exerting physiological activities. ${ }^{12} 13$ Originally, elevated galectin-3BP expression was more likely to be OW and OB. Galectin-3BP was positively related to OW or OB compared with NW. Some studies have reported that galectin-3BP is related to BMI and potentially OB in adults, ${ }^{18} 1930$ Furthermore, they also show that galectin-3BP is positively associated with the lipoprotein level. ${ }^{18}{ }^{19}$ Challa $e t a l^{31}$ reported that several adipokines are able to regulate the differentiation of adipocytes, and knockdown of galectin-3BP in preadipocyte cell lines increases adipocyte differentiation. Taken together with our results, galectin-3BP may also affect proliferation or other functions other than differentiation in adipose tissue, then influence human OB. Galectin-3BP might be a novel biomarker related to OB.

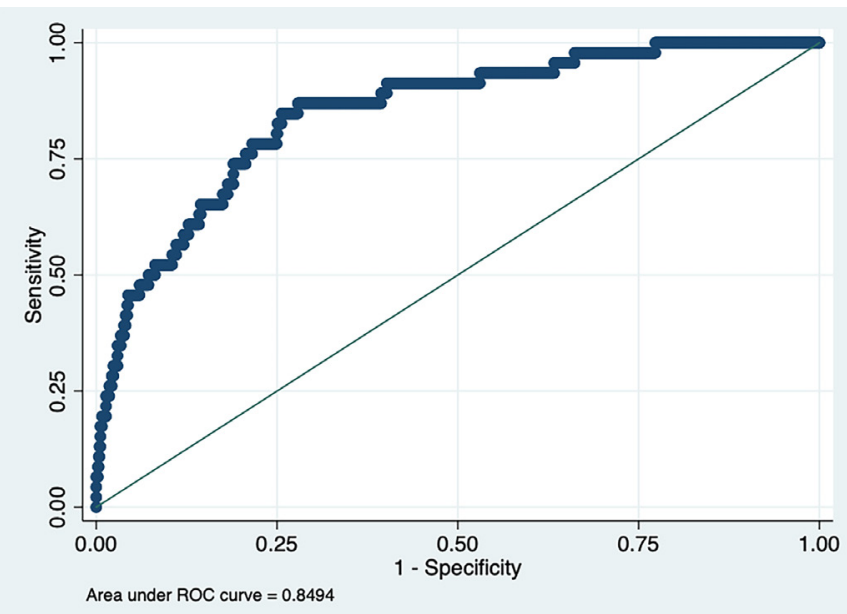

Figure 3 ROC curves of tertiles of galectin-3BP level for MetS. The area under the ROC curve was 0.85 (95\% Cl 0.79 to 0.91 ). Galectin-3BP, galectin-3 binding protein; MetS, metabolic syndrome; ROC, receiver operating characteristic.
Nonetheless, the precise activity and functions during metabolic control should be elaborated in future studies.

MetS is diagnosed when central OB plus any two of four other factors; however, the association between galectin-3BP level and MetS risk factors is still unknown. In the present study, we reported that the galectin-3BP level had a positive association with MetS among adolescents. Some mechanisms may account for such a relationship. First, galectin-3BP is correlated with components of visceral fat and levels of lipoprotein. ${ }^{18} 1921$ In addition, Roelofsen $e t a l^{20}$ reported that galectin-3BP is secreted from visceral adipose tissues. Typically, the increase in visceral fat is verified to further boost insulin resistance ${ }^{32}$; thus, MetS is probably induced by insulin resistance caused by galectin-3BP. Second, Niinaga $e t a l^{21}$ had confirmed the association between galectin-3BP and adiponectin through reconstituted proteins in vitro. Hypoadiponectinemia is closely associated with hypertension, dyslipidemia, diabetes mellitus, and visceral fat $\mathrm{OB}$ related to MetS. ${ }^{21} 33$ Third, inflammation is also a mechanism to account for such associations. Galectin-3BP is implicated in inflammatory distress, immune response $^{15}$ and chronic low-grade inflammation. ${ }^{21}$ It is widely reported that inflammation is essential to the pathogenesis underlying MetS. ${ }^{34}{ }^{35}$ Galectin-3BP is significantly positively associated with inflammatory markers, including interleukin (IL)-6, IL-1 $\beta$, and tumor necrosis factor alpha, ${ }^{19} 3637$ and together participate in MetS pathogenesis related to galectin-3BP, since inflammation may probably result in insulin resistance. Finally, galectin-3BP refers to a large oligomeric glycoprotein, and it has been recognized to be the galectin-3 ligand..$^{38}$ A previous study showed that the galectin-3 level is correlated with visceral fat, lipoprotein level, glucose homeostasis and even MetS. ${ }^{39} 40$ Therefore, it is possible that galectin-3BP may affect the distribution of body fat, gluconeogenesis, hyperglycemia, and lipolysis. Such an association leads to imbalanced lipid metabolism, while 
this may thereby result in a reduced capacity for maintaining the metabolic homeostasis.

Indeed, this study was the first to report an association between galectin-3BP and MetS in Chinese adolescents. Typically, we should not overlook the value of galectin-3BP in predicting the risk of MetS when used in combination with other biomarkers due to the low cost for detection. Nonetheless, certain limitations should also be noted in this study. First, the cross-sectional study design limited the ability to indicate the causality in the association. Therefore, prospective studies should be carried out for verifying the causality between MetS and galectin-3BP in adolescents. Second, all adolescents were enrolled from the same city, which might not necessarily represent the general Chinese adolescent population. Third, we did not use other adipokine (adiponectin or leptin) levels. We may compare galectin-3BP with adiponectin in terms of the ROC curve for MetS in the future. Finally, this work did not consider the pubertal stage among those recruited adolescents. Nevertheless, sex and age were adopted as the confounders when carrying out multiple logistic regression analysis, thus minimizing the impacts of the limitation.

This study suggests that elevated galectin-3BP expression might serve as a marker for predicting MetS among Chinese adolescents. In conclusion, findings in this study demonstrated that an elevated galectin-3BP was related to overweight adolescents and an even greater association with adolescents with OB. Galectin-3BP level had a positive association with MetS. This finding is particularly important for adolescents, who have an increasing risk for $\mathrm{OB}$ and MetS. Galectin-3BP can predict the risk of MetS when used in combination with other biomarkers due to its low cost for detection. Nonetheless, more studies should be carried out to clarify the galectin-3BP role as a biomarker in MetS.

\section{Acknowledgements The authors thank the participants in the study.}

Contributors YM and DW conceived and designed the study. SZ, YH, ZZ and XY collected, managed, and analyzed the data. SZ and YH drafted the manuscript. All authors read and approved the final version of the manuscript.

Funding This work was supported by the National Natural Science Foundation of China (71774173).

\section{Competing interests None declared.}

Patient consent for publication Not required.

Ethics approval The study was approved by the ethics committee of China Medical University ((2017) 055).

Provenance and peer review Not commissioned; externally peer reviewed.

Data availability statement Data are available upon reasonable request.

Supplemental material This content has been supplied by the author(s). It has not been vetted by BMJ Publishing Group Limited (BMJ) and may not have been peer-reviewed. Any opinions or recommendations discussed are solely those of the author(s) and are not endorsed by BMJ. BMJ disclaims all liability and responsibility arising from any reliance placed on the content. Where the content includes any translated material, BMJ does not warrant the accuracy and reliability of the translations (including but not limited to local regulations, clinical guidelines, terminology, drug names and drug dosages), and is not responsible for any error and/or omissions arising from translation and adaptation or otherwise.
Open access This is an open access article distributed in accordance with the Creative Commons Attribution Non Commercial (CC BY-NC 4.0) license, which permits others to distribute, remix, adapt, build upon this work non-commercially, and license their derivative works on different terms, provided the original work is properly cited, appropriate credit is given, any changes made indicated, and the use is non-commercial. See: http://creativecommons.org/licenses/by-nc/4.0/.

ORCID iD

Shihan Zhen http://orcid.org/0000-0003-3248-2684

\section{REFERENCES}

1 Zhou Y, Zhang Q, Wang T, et al. Prevalence of overweight and obesity in Chinese children and adolescents from 2015. Ann Hum Biol 2017;44:642-3.

2 Guo Y, Yin X, Wu H, et al. Trends in overweight and obesity among children and adolescents in China from 1991 to 2015: a metaanalysis. Int J Environ Res Public Health 2019;16:4656.

3 Liang Y, Hou D, Zhao X, et al. Childhood obesity affects adult metabolic syndrome and diabetes. Endocrine 2015;50:87-92.

4 Sasya M, Devi KSS, Babu JK, et al. Metabolic Syndrome-An emerging constellation of risk factors: electrochemical detection strategies. Sensors 2019;20:103.

5 Ye P, Yan Y, Ding W, et al. [Prevalence of metabolic syndrome in Chinese children and adolescents: a Meta-analysis]. Zhonghua Liu Xing Bing Xue Za Zhi 2015;36:884-8.

6 Fu J, Prasad HC. Changing epidemiology of metabolic syndrome and type 2 diabetes in Chinese youth. Curr Diab Rep 2014;14:447.

7 Stocks T, Bjørge T, Ulmer H, et al. Metabolic risk score and cancer risk: pooled analysis of seven cohorts. Int J Epidemiol 2015;44:1353-63.

8 Miao Z, Wang J, Wang F, et al. Comparative proteomics of umbilical vein blood plasma from normal and gestational diabetes mellitus patients reveals differentially expressed proteins associated with childhood obesity. Proteomics Clin Appl 2016;10:1122-31.

9 Moulder R, Bhosale SD, Goodlett DR, et al. Analysis of the plasma proteome using ITRAQ and TMT-based isobaric labeling. Mass Spectrom Rev 2018;37:583-606.

10 Savedoroudi P, Bennike TB, Kastaniegaard K, et al. Serum proteome changes and accelerated reduction of fat mass after laparoscopic gastric plication in morbidly obese patients. J Proteomics 2019;203:103373.

11 Wang Y, Xu LY, Lam KSL, et al. Proteomic characterization of human serum proteins associated with the fat-derived hormone adiponectin. Proteomics 2006;6:3862-70.

12 Nonaka M, Ma BY, Imaeda H, et al. Dendritic cell-specific intercellular adhesion molecule 3-grabbing non-integrin (DC-SIGN) recognizes a novel ligand, Mac-2-binding protein, characteristically expressed on human colorectal carcinomas. J Biol Chem 2011;286:22403-13.

13 White MJV, Roife D, Gomer RH. Galectin-3 binding protein secreted by breast cancer cells inhibits monocyte-derived fibrocyte differentiation. J Immunol 2015;195:1858-67.

14 Dumic J, Dabelic S, Flögel M. Galectin-3: an open-ended story. Biochim Biophys Acta 2006;1760:616-35.

15 Cai H, Lu S, Chen Y, et al. Serum retinol binding protein 4 and galectin-3 binding protein as novel markers for postmenopausal nonalcoholic fatty liver disease. Clin Biochem 2018;56:95-101.

16 Lin T-W, Chang H-T, Chen C-H, et al. Galectin-3 binding protein and galectin-1 interaction in breast cancer cell aggregation and metastasis. J Am Chem Soc 2015;137:9685-93.

17 Koths K, Taylor E, Halenbeck R, et al. Cloning and characterization of a human Mac-2-binding protein, a new member of the superfamily defined by the macrophage scavenger receptor cysteine-rich domain. J Biol Chem 1993;268:14245-9.

18 Sugiura T, Dohi Y, Takase H, et al. Serum levels of Mac-2 binding protein increase with cardiovascular risk and reflect silent atherosclerosis. Atherosclerosis 2016;251:192-6.

19 Gleissner CA, Erbel C, Linden F, et al. Galectin-3 binding protein, coronary artery disease and cardiovascular mortality: insights from the LURIC study. Atherosclerosis 2017;260:121-9.

20 Roelofsen H, Dijkstra M, Weening D, et al. Comparison of isotopelabeled amino acid incorporation rates (CILAIR) provides a quantitative method to study tissue secretomes. Mol Cell Proteomics 2009;8:316-24.

21 Niinaga R, Yamamoto H, Yoshii M, et al. Marked elevation of serum M2BP-adiponectin complex in men with coronary artery disease. Atherosclerosis 2016;253:70-4.

22 Han Y, Ma Y, Liu Y, et al. Plasma cholinesterase is associated with Chinese adolescent overweight or obesity and metabolic syndrome prediction. Diabetes Metab Syndr Obes 2019;12:685-702. 
23 Subspecialty Group of Endocrinologic, Hereditary and Metabolic Diseases, The Society of Pediatrics, Chinese Medical Association, Subspecialty Group of Cardiology, The Society of Pediatrics, Chinese Medical Association, Subspecialty Groups of Child Health Care, The Society of Pediatrics, Chinese Medical Association. [The definition of metabolic syndrome and prophylaxis and treatment proposal in Chinese children and adolescents]. Zhonghua $\mathrm{Er} \mathrm{Ke} \mathrm{Za}$ Zhi 2012;50:420-2.

24 Han Y, Liu Y, Zhao Z, et al. Does physical activity-based intervention improve systemic proinflammatory cytokine levels in overweight or obese children and adolescents? insights from a meta-analysis of randomized control trials. Obes Facts 2019;12:653-68.

$25 \mathrm{Hu}$ C. Aldehyde dehydrogenases genetic polymorphism and obesity: from genomics to behavior and health. Adv Exp Med Biol 2019;1193:135-54.

26 Perillo NL, Marcus ME, Baum LG. Galectins: versatile modulators of cell adhesion, cell proliferation, and cell death. $\mathrm{J} \mathrm{Mol} \mathrm{Med}$ 1998;76:402-12.

27 Kiwaki K, Novak CM, Hsu DK, et al. Galectin-3 stimulates preadipocyte proliferation and is up-regulated in growing adipose tissue. Obesity 2007;15:32-9.

28 Baek J-H, Kim S-J, Kang HG, et al. Galectin-3 activates PPAR $\gamma$ and supports white adipose tissue formation and high-fat diet-induced obesity. Endocrinology 2015;156:147-56.

29 Rhodes DH, Pini M, Castellanos KJ, et al. Adipose tissue-specific modulation of galectin expression in lean and obese mice: evidence for regulatory function. Obesity 2013;21:310-9.

30 Gleissner CA, Erbel C, Linden F, et al. Galectin-3 binding protein plasma levels are associated with long-term mortality in coronary artery disease independent of plaque morphology. Atherosclerosis 2016;251:94-100.
31 Challa TD, Straub LG, Balaz M, et al. Regulation of de novo adipocyte differentiation through cross talk between adipocytes and preadipocytes. Diabetes 2015;64:4075-87.

32 Hayashi T, Boyko EJ, McNeely MJ, et al. Visceral adiposity, not abdominal subcutaneous fat area, is associated with an increase in future insulin resistance in Japanese Americans. Diabetes 2008;57:1269-75.

33 Matsuzawa Y, Funahashi T, Kihara S, et al. Adiponectin and metabolic syndrome. Arterioscler Thromb Vasc Biol 2004;24:29-33.

34 Wisse BE. The inflammatory syndrome: the role of adipose tissue cytokines in metabolic disorders linked to obesity. J Am Soc Nephrol 2004;15:2792-800.

35 Rochlani Y, Pothineni NV, Kovelamudi S, et al. Metabolic syndrome: pathophysiology, management, and modulation by natural compounds. Ther Adv Cardiovasc Dis 2017;11:215-25.

36 Maekawa T, Kamada Y, Ebisutani Y, et al. Serum Mac-2 binding protein is a novel biomarker for chronic pancreatitis. World $J$ Gastroenterol 2016;22:4403-10.

37 Gagno G, Padoan L, Stenner E, et al. Galectin 3 and galectin 3 binding protein improve the risk stratification after myocardial infarction. J Clin Med 2019;8:570.

38 Grassadonia A, Tinari N, lurisci I, et al. 90K (Mac-2 BP) and galectins in tumor progression and metastasis. Glycoconj $J$ 2002;19:551-6.

39 Nayor M, Wang N, Larson MG, et al. Circulating galectin-3 is associated with cardiometabolic disease in the community. J Am Heart Assoc 2015;5:e002347.

40 Pugliese G, lacobini C, Pesce CM, et al. Galectin-3: an emerging all-out player in metabolic disorders and their complications. Glycobiology 2015;25:136-50. 